\title{
BEHAVIOUR BASED MOBILE ROBOT NAVIGATION TECHNIQUE USING AI SYSTEM: EXPERIMENTAL INVESTIGATION ON ACTIVE MEDIA PIONEER ROBOT
}

\author{
S. Parasuraman ${ }^{1}$, V. Ganapathy $^{2}$, BiJan Shirinzadeh ${ }^{3}$ \\ ${ }^{1,2}$ Monash University Malaysia, Bandar Sunway, Malaysia \\ ${ }^{3}$ Monash University, Clayton, Victoria, Australia, \\ s.parasuraman@eng.monash.edu.my
}

\begin{abstract}
A}$ key issue in the research of an autonomous robot is the design and development of the navigation technique that enables the robot to navigate in a real world environment. In this research, the issues investigated and methodologies established include (a) Designing of the individual behavior and behavior rule selection using Alpha level fuzzy logic system (b) Designing of the controller, which maps the sensors input to the motor output through model based Fuzzy Logic Inference System and (c) Formulation of the decision-making process by using Alpha-level fuzzy logic system. The proposed method is applied to Active Media Pioneer Robot and the results are discussed and compared with most accepted methods. This approach provides a formal methodology for representing and implementing the human expert heuristic knowledge and perception-based action in mobile robot navigation. In this approach, the operational strategies of the human expert driver are transferred via fuzzy logic to the robot navigation in the form of a set of simple conditional statements composed of linguistic variables.
\end{abstract}

Keywards: Mobile robot, behavior based control, fuzzy logic, alpha level fuzzy logic, obstacle avoidance behavior and goal seek behavior

\section{INTRODUCTION}

The real world environment during mobile robot navigation has the following problems: a) Knowledge of the environment is partial, uncertain, imprecise and approximate, b) The environment is vast and dynamic and the obstacles can move, appear or disappear and c) Due to the quality of the ground, sensors data received are not completely reliable. The issues (a) and (b) affect the behavior rule selection and (c) affects the sensors input space to match the complex environment into robot's output. In the past, several works relating to robot navigation have been done which describe mathematical models and fuzzy logic systems for behavior selection, but the limitations are the insufficient knowledge based perception of the environment and absence of decision-making capability similar to human driver.

A variety of behavior-based control schemes have been inspired by the success of Brooks work [1]. Arkin [2] has described the use of reactive behaviors called schemas. In this method, potential field is used to define the output of each schema. Then all the outputs are combined by weighted summation. Similar techniques for defining reactive control behaviors are used by Gat [3], Anderson and Donath [4] and Tianmio and Bo [5]. The adaptation of the action selection problem given in [6] is constrained to a particular mathematical model.

P. Althauas et al., [7] have described a scheme for behavior coordination that is based on a non-linear dynamical system approach. Coordinations among behaviors are modeled by 
means of an additional competitive dynamics that controls the weights of behaviors. This method also encounters the problem of multiple behavior conflicts between the outputs of different concurrent behaviors. Coordination mechanism suggested in [8] and [9] is called a temporal sequencing, in which the system is partitioned into discrete operating states and enumerates the perceptual trigger, which causes transitions between those states. Examples of cooperative mechanisms are the potential-field method [10], vector summation used in [11], blending of fuzzy behaviors [12], the dynamic system approach [13] and voting mechanism [14].

The authors of [6] to [14] describe the mobile robot navigation techniques using mathematical models for behavior selection and they do not deal with the natural way of describing the behavior rules and behavior selection during behavior conflicts.

Behavior arbitration scheme introduced by Saffiotti et al., [15] used fuzzy logic system, which allows one behavior at a time to be active. Another technique [16] focuses on combining the input of each behavior using predetermined weighting factors. This leads to direct conflict in execution when multiple behaviors give contradictory commands. To deal with these limitations, another strategy called fusion methodology is employed in which each behavior is allowed to provide the final output based on the situational context, [15] and [17]. Tunstel et al., [17] have used a similar method of [15], in that, adaptive hierarchies of multiple fuzzy behaviors are combined and the output is actuated based on the degree of applicability. In this approach, certain behaviors are allowed to influence the overall behaviors based on the current situation and goal. In order to perform realistic tasks of a mobile robot in a complex surrounding, it must select 'the most appropriate' or 'the most relevant' next action at a particular moment, when facing a particular problem.

In this paper, behavior rule selection or decision-making procedure incorporating Alphalevel fuzzy logic system is discussed and used for selecting an appropriate action during mobile robot navigation. In the proposed approach, the control model is also presented incorporating the Alpha level fuzzy logic system. In the present approach, the operational strategies of the human expert driver are transferred via fuzzy logic to the robot navigation in the form of optimized behavior rules and resolved the conflicts similar to human way of decision making capabilities. The technique presented in this paper is validated by conducting simulation and real world experiments using Active Media Pioneer Robot.

\section{THEORETICAL WORK}

\subsection{Behavior rules conflict and Alpha-level thresholds}

When the context data of the environment are matched with the condition parts of the logic rules in a rule base of the robot navigation system, it may be possible that more than one rule is fired. The set of rules fired in this manner is termed as the conflicts set. Then, a method of conflict resolution must be invoked to select the rule that would be fired. Conflict resolution in the control of behavior-based robot is the control decision process, which should be taken as a result of the firing among several similar kinds of fuzzy behavior rules. In the present mobile robot system, control of a robot is shared between a set of perception based action units, called behaviors. These behaviors are obstacle avoidance, seek goal, wall follow, terrain etc. Behavior rule selection in robot navigation is the way that an agent selects the most appropriate or the most relevant next action to take in a particular moment, when facing a particular problem. Based on environment context data, each behavior produces an action to control the robot with respect to a particular objective such as avoid obstacle, goal seek etc. When sensory data matches several behavior rules (condition part of the rules), then more than one behavior rule is fired at a time. As a result of multiple rule firing, behavior rules 
conflict each other. In order to resolve these conflicts, an Alpha level fuzzy inference system is introduced and this establishes the minimum truth threshold for a particular behavior rule that needs to be fired at a time. As and example "If s1 is small and s2 is small then the output is Alphathreshold value". The truth of the fuzzy proposition ' $s 1$ is small' is less than [0.3], this rule will not fire due to the set level of Alpha limit. Thus an Alpha threshold is an important control in the execution of fuzzy rules. Behaviors within the environment are well defined by a set of control parameters through fuzzy sets and fuzzy membership values.

\subsection{Structure of controller with Alpha threshold level}

The overall structure of the PID controller interfaced with the Fuzzy Inference System (FIS) with Alpha threshold level is shown in Fig. 1 and the block diagram shows that the mobile robot (each wheel drives a separate motor) is controlled by a PID controller and the controller is executed in an interrupt routine of the main processor. The motor controller is used in two control modes: speed and position modes. Figure 1 schematically shows the location of Alpha threshold level in the fuzzy control system. The Alpha threshold stored in the system control block (XSYSalfacut) is the primary threshold value in the fuzzy system and is initiated to zero when the fuzzy model is connected to the control system (MdlConnecttoFMS). This value is automatically used by the fuzzy conditional proposition (FzyCondProposition) and fuzzy unconditional proposition (FzyUnCondProposition) to decide whether or not the predicate is true. When fuzzy sets and fuzzy variables are initialised, their alpha thresholds are inherited from the current output value of system control block (XSYSalfacut). The alpha cut stored with fuzzy set descriptor block $(F D B)$ is used to control the truth membership array resulting from the fuzzy set operators. This value inherited from the threshold associated with the variable (VDBalfacut) is used to adjust the solution prior to defuzzification [18]. The Alpha level fuzzy logic methodology is established mathematically, analysed and discussed in the reference [18].

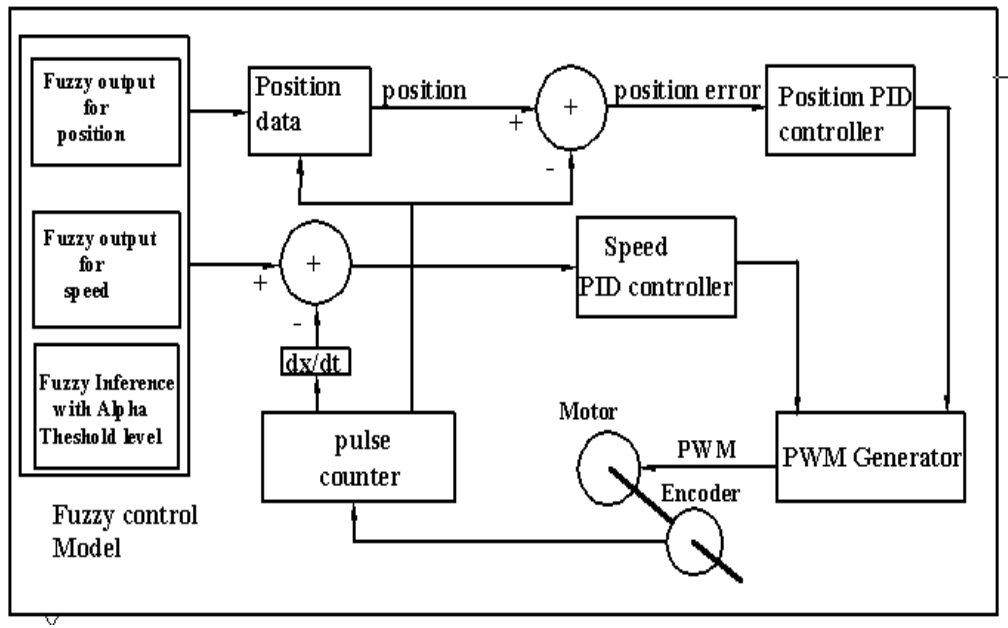

Fig 1. Control model of a mobile robot with Fuzzy Inference System (FIS). 


\section{EXPERIMENTAL WORK}

\subsection{Modeling the control parameters}

The Robot behaviors tested by using the present method comprise of Obstacle avoidance, Seek goal and wandering behaviors. All these behaviors are developed for Active Media Pioneer Robot, which is shown in Fig. 2. Active Media Pioneer Robot uses the front sonar arrays with eight transducers that provide object detection around obstacles (Fig. 2(a)). The sonar positions in all arrays are fixed one on each side, and six facing outwards at 20-degree intervals as shown in Fig. 2(a). These sensors report the distances between the robot and the closest obstacle in each of the eight sectors (0-7). Each obstacle distance is represented by the three linguistic input fuzzy sets $\{$ small, medium, big $\}$ and two output fuzzy sets which are speed and turn angle. The input and output fuzzy sets are as shown in the Fig. 3 (a) and (b).

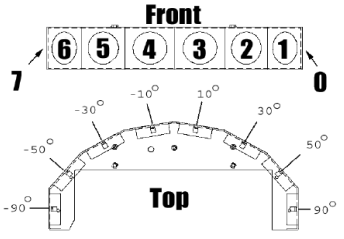

(a)

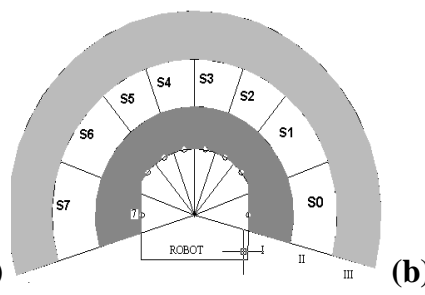

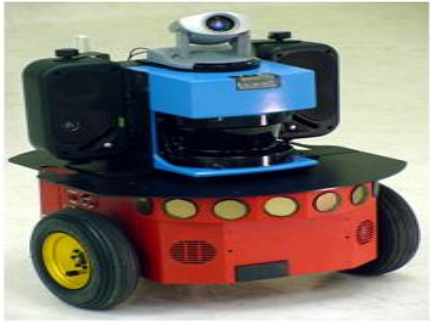

(c)

Fig. 2: Active Media Pioneer Robot (a) Sensors Position (b) Sensors region (c) Actual robot.

Input parameters, which provide the context data of the environments, are the front sensors (S3 and S4) data are shown in Fig. 2(b). These data are converted into three fuzzy sets namely small, medium and big. The small fuzzy set is further sub-divided into Alpha level fuzzy set as shown in Fig. 3 (a). Similarly the output data is converted into five number of fuzzy sets namely Medium Positive (MP), Small Positive (SP), zero (Z), Small Negative $(S N)$ and Medium Negative $(M N)$. The output fuzzy sets are further sub-divided into Alpha level sets as shown in Fig. 3 (b).
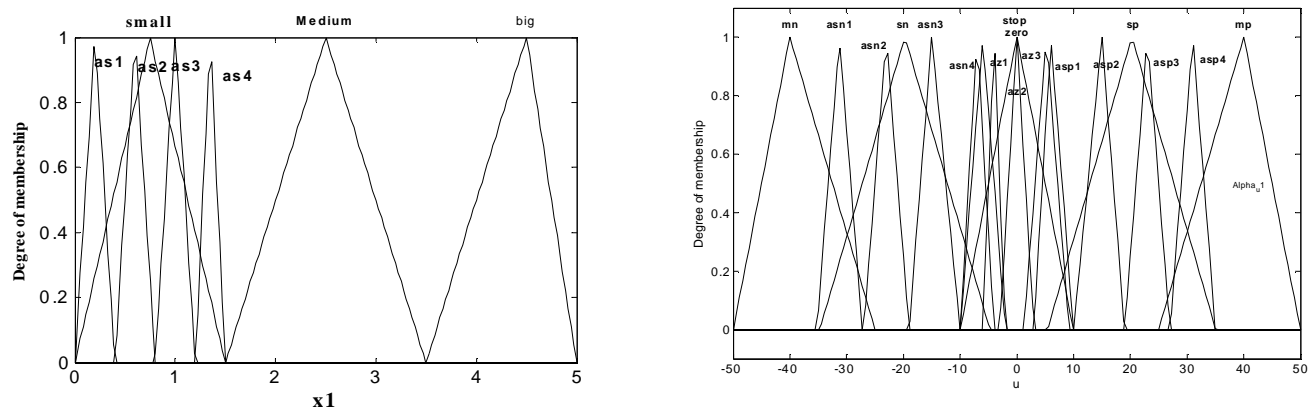

Fig. 3 (a). Input fuzzy set with alpha intervals. (b). Fuzzy output set with alpha intervals.

Behaviors rules are established using the alpha level intervals of input and output fuzzy sets based on the environment perceived by sensors $S 3$ and $S 4$. 


\subsection{Behavior Selection}

Figure 4 shows the FIS for behavior rule selection based on the Alpha threshold level set in the input and output fuzzy sets. Figure 5 shows an example of rule firing based on the proposed methodology.

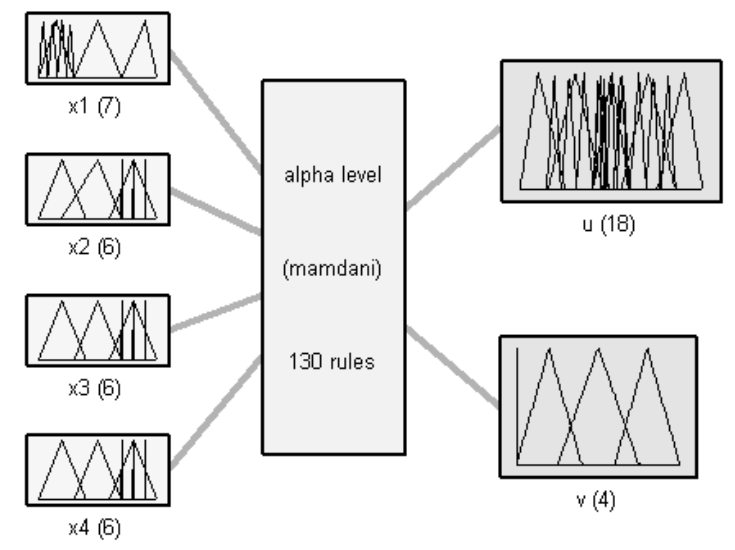

System alpha level: 4 inputs, 2 outputs, 130 rules

Fig. 4:. FIS for behavior selection.

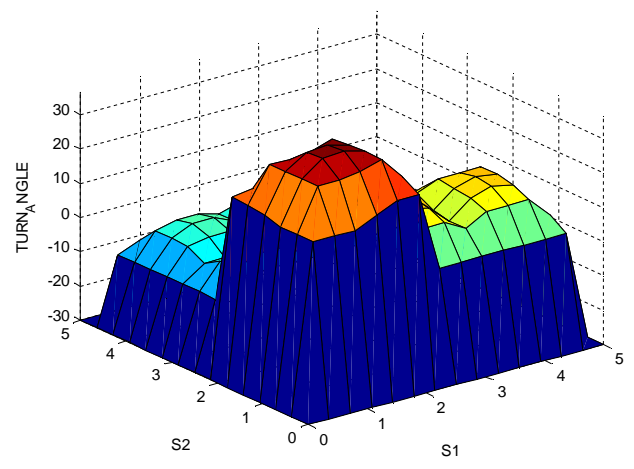

Fig. 5: Rule firing during Navigation.

\subsection{Avoid- obstacle behavior}

This behavior is to allow the robot to avoid collision with obstacles while it maintains its navigation. It is a combination of three simple behaviors namely Move, Turn and Stop. The decision of each behavior depends on the behavior rules, which are established based on proposed methodology. Examples of some of the rules are shown below:

110. If ( $\mathrm{x} 2$ is not as3) and ( $\mathrm{x} 3$ is Alpha3) and ( $\mathrm{x} 4$ is Alpha2) and ( $\mathrm{x} 5$ is not Alpha1) then ( $\mathrm{u}$ is Alpha_u1)(v is low) (0)

111. If ( $\mathrm{x} 2$ is not as 3$)$ and ( $\mathrm{x} 3$ is Alpha1) and ( $\mathrm{x} 4$ is Alpha2) and ( $\mathrm{x} 5$ is not Alpha1) then ( $\mathrm{u}$ is az1)(v is low) (0)

112. If ( $x 2$ is not as3) and ( 33 is Alpha1) and ( $x 4$ is Alpha_3) and (x5 is not Alpha1) then ( $\mathrm{u}$ is az1)( $\mathrm{v}$ is low) (0)

113. If ( $\mathrm{x} 2$ is not as3) and ( $\mathrm{x} 3$ is Alpha3) and ( $\mathrm{x} 4$ is Alpha1) and ( $\mathrm{x} 5$ is not Alpha1) then ( $\mathrm{u}$ is Alpha_u1)(v is low) (0) 
114. If ( $\mathrm{x} 2$ is not as3) and ( $\mathrm{x} 3$ is Alpha3) and ( $\mathrm{x} 4$ is Alpha2) and ( $\mathrm{x} 5$ is not Alpha1) then (u is Alpha_u1)(v is low) (0)

115. If ( $x 2$ is not as3) and ( $x 3$ is Alpha3) and ( $x 4$ is Alpha_3) and ( $x 5$ is not Alpha1) then (u is $\mathrm{az} 1)(\mathrm{v}$ is low) $(0)$

116. If ( $\mathrm{x} 2$ is not as3) and ( $\mathrm{x} 3$ is Alpha2) and ( $\mathrm{x} 4$ is Alpha1) and ( $\mathrm{x} 5$ is not Alpha1) then ( $\mathrm{u}$ is Alpha_u1)(v is low) (0)

117. If ( $\mathrm{x} 2$ is not as3) and ( $\mathrm{x} 3$ is Alpha2) and ( $\mathrm{x} 4$ is Alpha2) and ( $\mathrm{x} 5$ is not Alpha1) then ( $\mathrm{u}$ is Alpha_u1)(v is low) (0)

\subsection{Goal-attract}

A set of fuzzy logic navigation rules that drive the robot from a known initial position to a user-specified goal position are presented as shown below:

IF $\delta$ is goal far-left, THEN $\omega$ is large-neg

IF $\delta$ is goal medium-left, THEN $\omega$ is medium-neg

IF $\delta$ is goal-left, THEN $\omega$ is small-neg

IF $\delta$ is goal head-on, THEN $\omega$ is zero

IF $\delta$ is goal right, THEN $\omega$ is small-pos

IF $\delta$ is goal medium-right, THEN $\omega$ is medium-pos

IF $\delta$ is goal far- right, THEN $\omega$ is large-pos

Where $\delta$ is the goal direction and $\omega$ is the turn angle of the robot. In these cases, the robot initially performs an in-place rotation towards the goal to nullify the heading error. Once the robot is aligned with the goal direction, then it proceeds towards the goal position on a straight path.

\subsection{Control Model}

Control Model $e(u)$ is established and cited in the reference [18] and also shown below $e(u)=\min \left[\mu_{\alpha}(u), \mu_{C}(u)\right]$.

where

$$
\begin{aligned}
& \mu_{C}(u)=e_{\max }\left[\left(\mu_{R_{i}\left(X_{1}, X_{2}, \ldots X_{i} \ldots X_{n}\right)}\right)\right] \\
& \mu_{\alpha}(u)=e_{\max }\left[\left(\mu R_{i}\left(X_{1}^{\prime}, X_{2}^{\prime}, \ldots \ldots X_{i}^{\prime} \ldots X_{n}^{\prime}\right)\right] .\right.
\end{aligned}
$$

and

where

$\mu_{\alpha}(u)$ and $\mu_{C}(u)$ are the outputs. The parameters in the equation 1 (a) and 1(b) are as defined in the reference [18].

\section{RESULTS AND DISCUSSION}

The Control model e(u) is applied to the mobile robot, and simulation and real world experiments are conducted. Based on the environmental context, the output is selected between $\mu_{\alpha}(u)$ and $\mu_{C}(u)$. The results are analysed and compared with the most accepted methods.

\subsection{Simulation}

Simulation is conducted at two different situations, which are discussed in this section and the simulation results are shown in Figs. 6 to 13. The simulation environment is built with maps, which represent obstacles, home position of robot and the robot with sonar sensors. Robot and sensors are designed based on the real parameters of Active Media 
Pioneer Robot. The maps, which are designed for navigation are unstructured and contain various objects with unsymmetrical shapes and sizes and placed in different locations. There are 14 sensors and each sensor array delivers the echo corresponding to the maximum distance of $5000 \mathrm{~mm}$, if there is no object in front of the sensor.

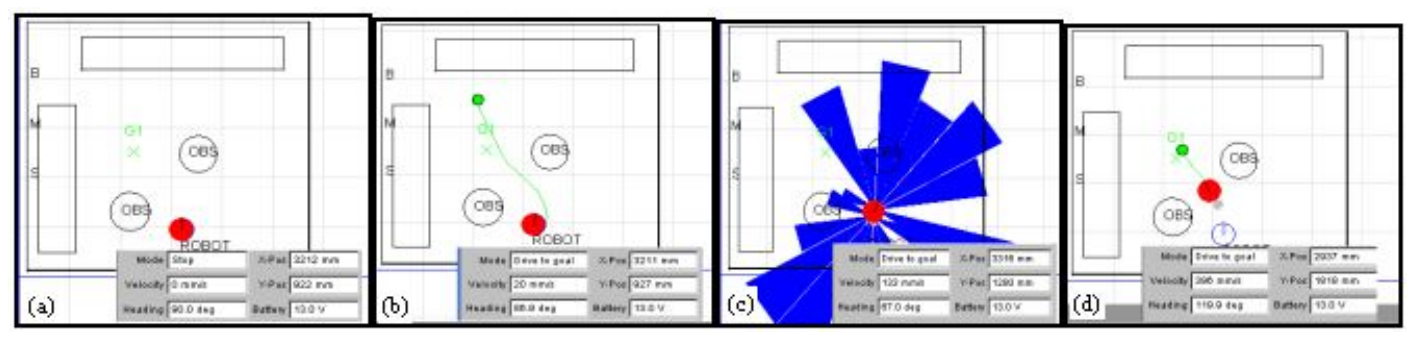

Fig. 6: Robot navigate towards goal while avoiding obstacle in the situation of environmental conflicts.

The experimental results shown in Fig.s 6(a-d) are the simulation results of Active Media Pioneer Robot. The results show the robot path from the initial position to the target position with various obstacles. The robot behavior rules in these positions in the present situation are conflicting because of multiple rules activation at the same time. In Fig. 6(a), the robot is facing two obstacles, which are located at a distance of 0.6 meter and 1.0 meter respectively from THE robot. These obstacles are located in the vicinity of the one fuzzy set, which is called small. In these situations, behavior rules conflict arises because more than one rules are active. This is due to more than one obstacle present in the small fuzzy set. In this situation, the Alpha level fuzzy logic rules are active for navigation with minimum proximity to the obstacles. Figure 6(b) shows the movement of the robot that is based on two different value of the Alpha level. In all situations, the robot decides its path based on the $\min$ value of the output membership functions $\mu_{\alpha}(u) \operatorname{or} \mu_{C}(u)$. Based on these control outputs, robot navigates in an optimum path and reaching the goal position, which are shown in Fig. 6(c) and Fig. 6(d).

The simulation results are evaluated as follows. Figure 7 shows the plot of sensors data S3 and S4 of the robot for a period of $300 \mathrm{sec}$. Plot illustrated in Fig. 8 is the turn angle plotted against distance traveled over a period of time $300 \mathrm{sec}$. In all these situations multiple obstacles are presented close to the robot. In these situations the simulation clearly indicates that the robot is turned and navigated without any behavior rule conflicts. From the graph it is observed that the robot navigates with a minimum deviation from each of the obstacle according to the Alpha intervals. This shows that the proposed approach provides better conflict resolution when compared to fuzzy behavior coordination approach used in the literature. The experimental results that are shown in Fig. 9 (a-d) are for the other situations, where more obstacles are placed. The results are analysed and plotted as shown in Fig. 10- 13. 


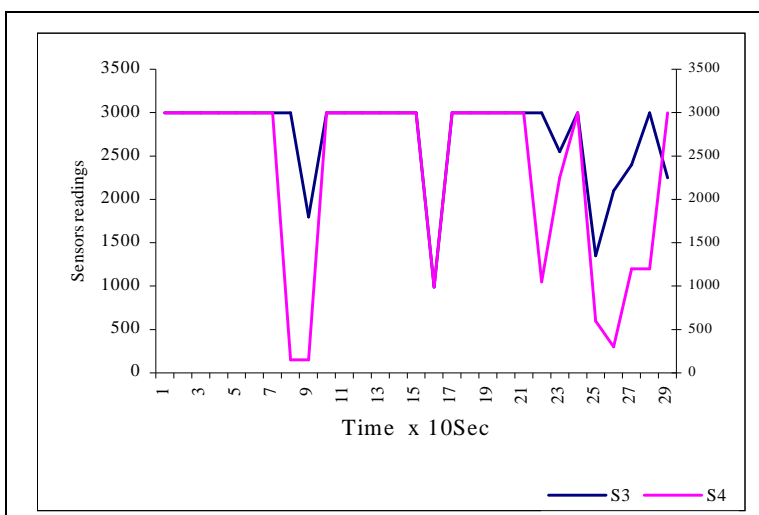

Fig 7: Sensors readings over a period of time $300 \mathrm{sec}$

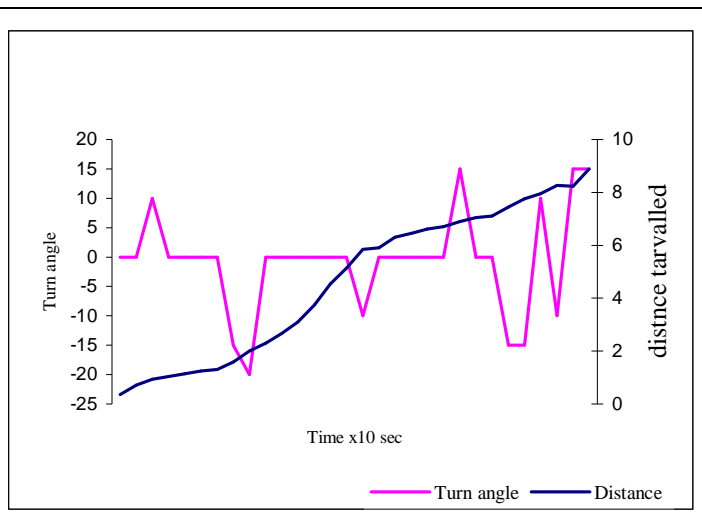

Fig. 8: Turn angle against travel distance over a period of time $300 \mathrm{sec}$.

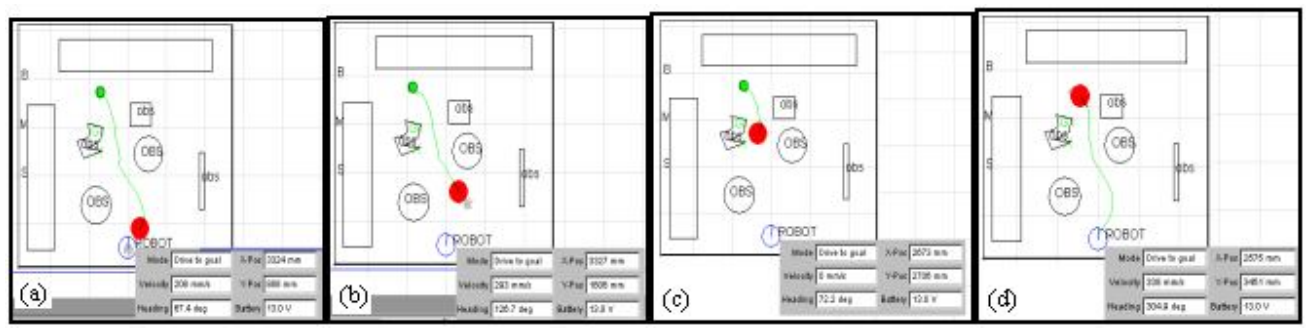

Fig 9: Robot navigates towards goal while avoiding obstacles in the situation of multiple behavior conflicts.

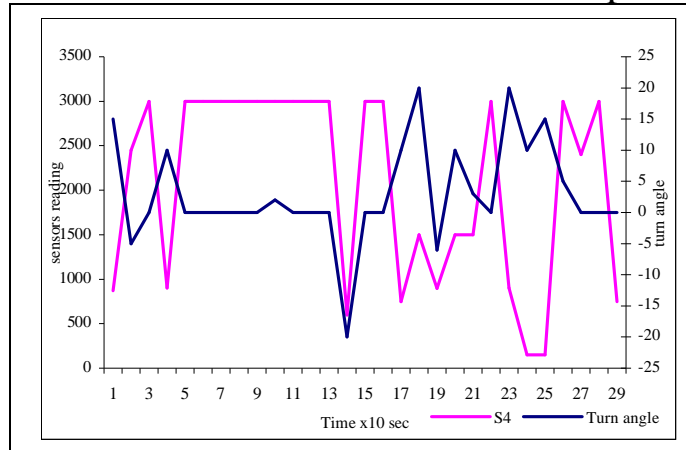

Fig 10: Sensors readings over a period of time $300 \mathrm{sec}$.

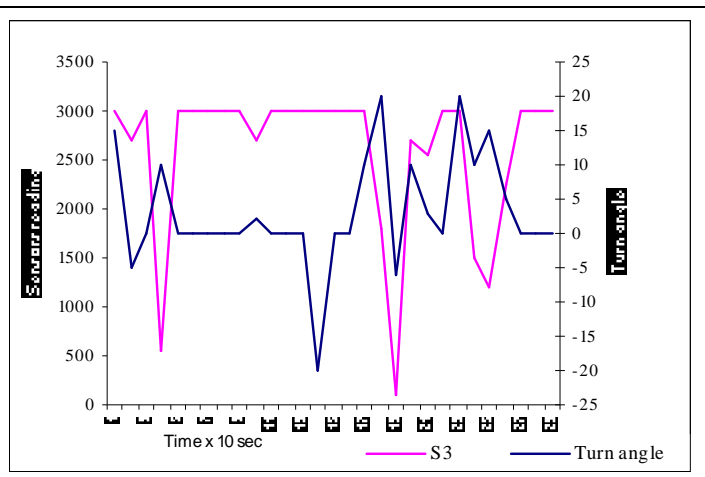

Fig. 11: Turn angle against travel distance over a period of time $300 \mathrm{sec}$.

The experiments conducted from the present study, it is concluded that, based on the multiple obstacles present close to the robot, the output turn angles are automatically computed based on the Alpha intervals and robot navigates with minimum deviation from obstacles. 


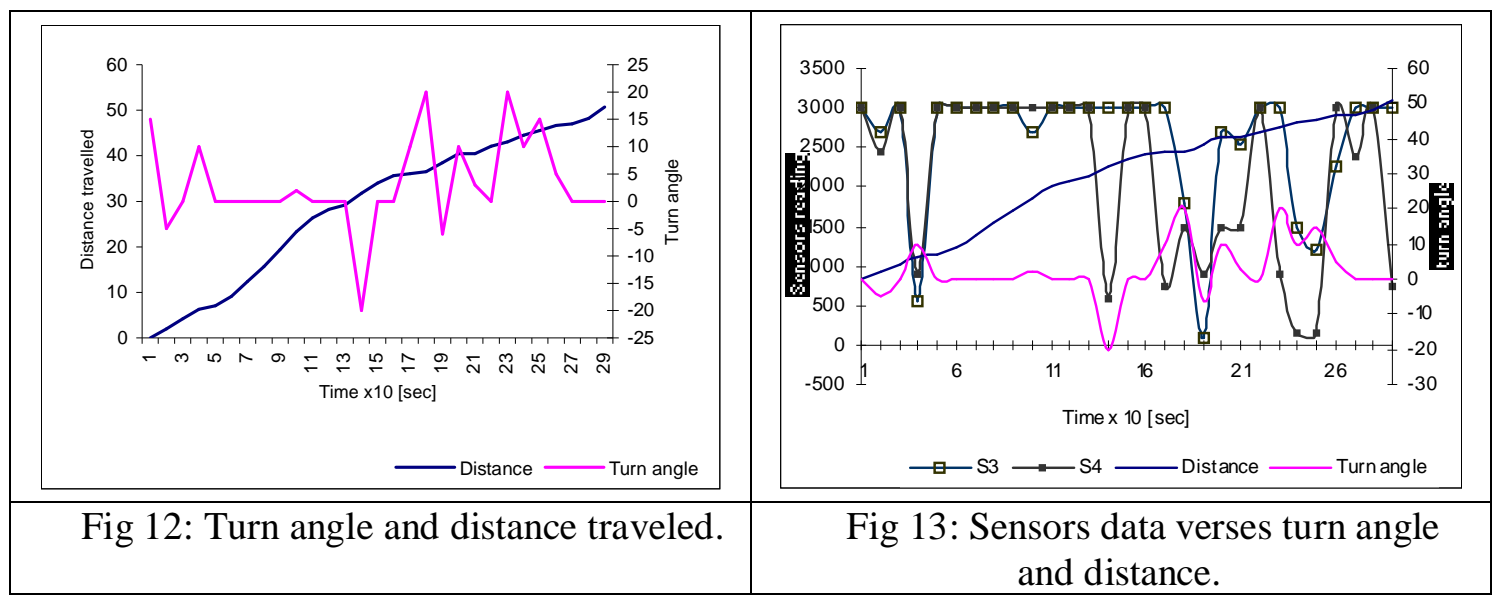

\subsection{Real World Experiments}

The behavior rules obtained from the proposed methodology are tested in a real world environment using Active Media Pioneer robot. Figure 14 shows various positions of the robot while encountering obstacles during navigation. Plot illustrated in Fig. 15 is the robot travel time plotted against sensors reading and turn angle over a time period of 300 sec. In this plot, it is observed that the robot's positions at $9^{\text {th }}$ and $22^{\text {nd }}$ sec, sensors S3 and $\mathrm{S} 4$ encounter obstacles each at a distance of $1000 \mathrm{~mm}$ from the robot. The distances from both sensors are inferenced as a small fuzzy set. In this situation Alpha level fuzzy threshold is active and the system decides the behavior rule selection. This is illustrated in the graph shown in Fig. 16. This figure illustrates the relationship between the obstacle positions from robot obtained by sensors S2 to S5 and the turn angle for a continuous run of $300 \mathrm{sec}$. From the real world experiments conducted using the present methodology, it is observed that the sensors' ( 2 2 to S5) data have been used and these data decide the robot turn angle based on fuzzy Alpha level intervals, when the robot faces an obstacle.

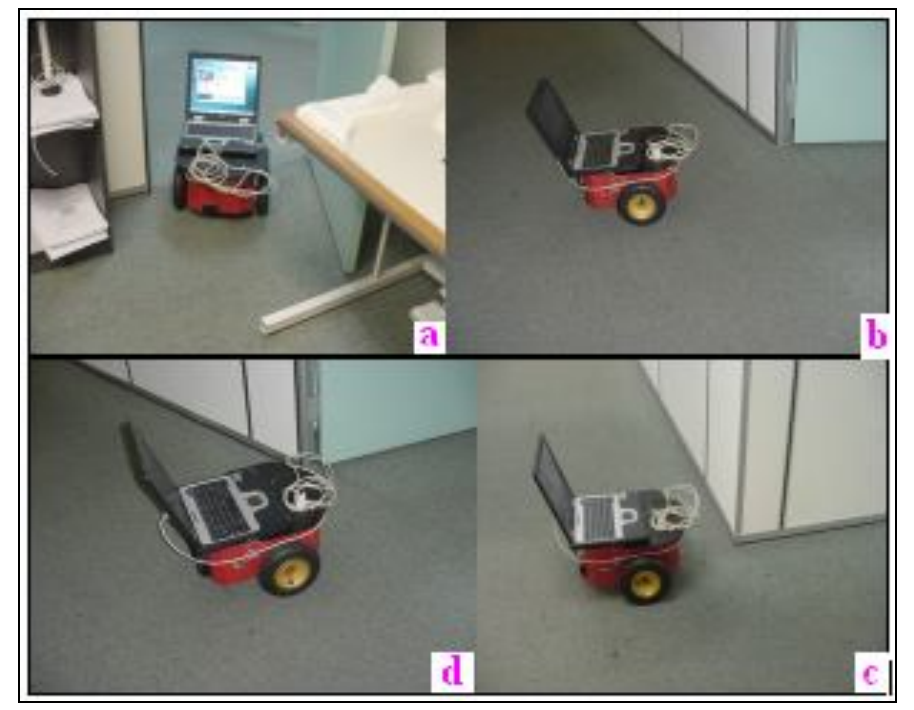

Fig. 14: (a), (b), (c) and (d): Active Media Pioneer Robot encountering walls and obstacles during navigation. 
In the proposed methodology, input and output fuzzy sets are set to minimum ranges using Alpha level fuzzy system so that the robots' turn angle deviations are minimum with respect to the obstacles positions in the environment. In the most accepted methods in the past, these values are set with larger intervals and as a result the angle of deviations from obstacles are quite large.

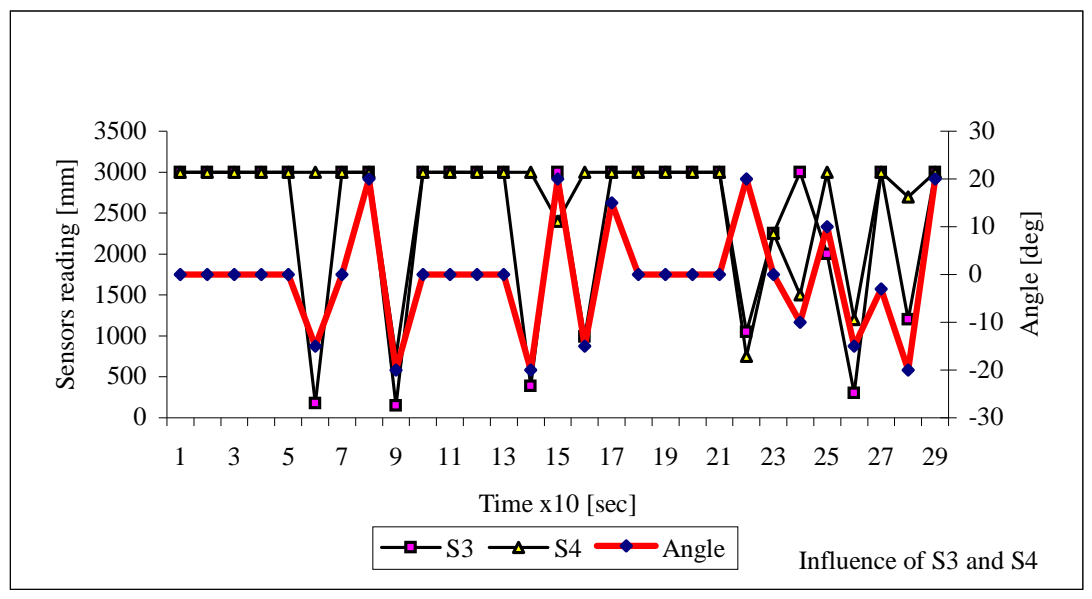

Fig.15: Plot of robot travelling time against output responses of the front sensors (S3S4) and turn angle of robot

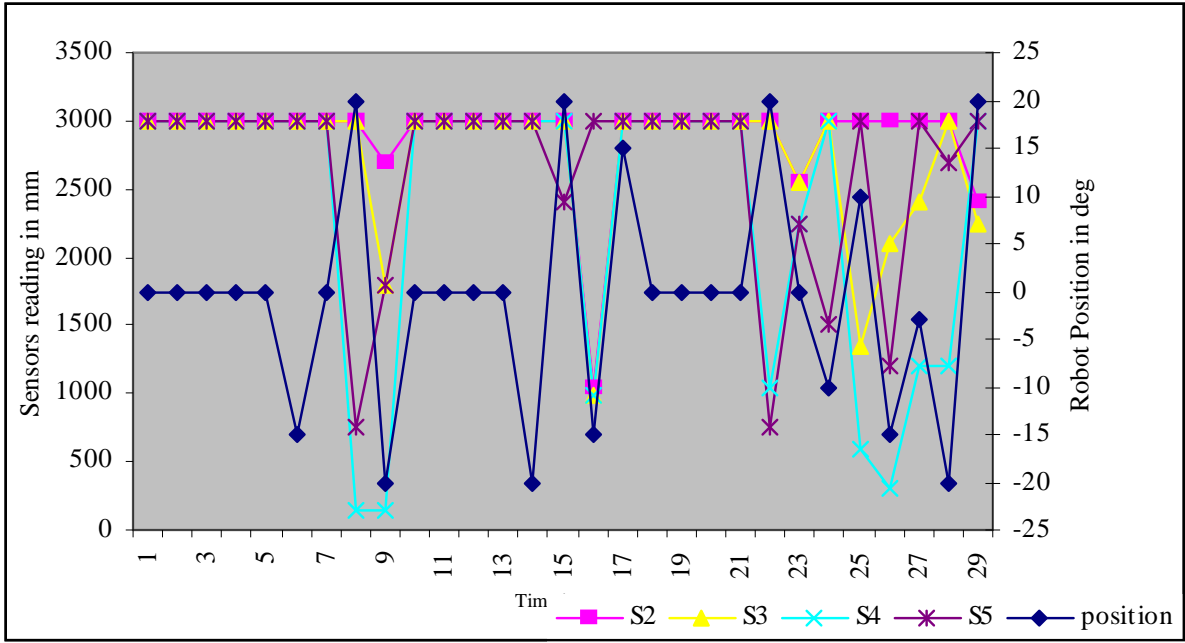

Fig. 16: Plot of robot travelling time against output responses of sensors (S2-S5) and turn angle of robot.

\section{5. . COMPARISONS}

Arkin's [2] motor schemas method is model based and the data perceptions are not dealt with the natural way of solving complex environment. The proposed methodology deals with a natural way of resolving behavior conflicts through Alpha level fuzzy logic system. Potential field methods [10] are constructed with a map-like internal representation of the world, while the proposed methodology of Alpha level fuzzy logic system does not need such a map. 
The robot navigation based on PID controller and Fuzzy logic system [22] is compared with the proposed Alpha fuzzy logic approach and the results are analysed using the plot shown in Fig. 17. In previous work given in [22], the PID and Fuzzy logic approaches used larger intervals of inputs and as a result the robot deviated much from obstacles through a wider angle during navigation. This affects the behavior selection, when more obstacles are present closely in the environment. In the proposed Alpha level fuzzy logic, inputs and output levels are set with Alpha level threshold limits and as a result the robot navigates with close proximity to obstacles with minimum deviation especially when the obstacles are present closely. In Fig. 17, the proposed Alpha level fuzzy system shows that the robot deviates with minimum angle from obstacles compared to the other two approaches used in the ref [22].

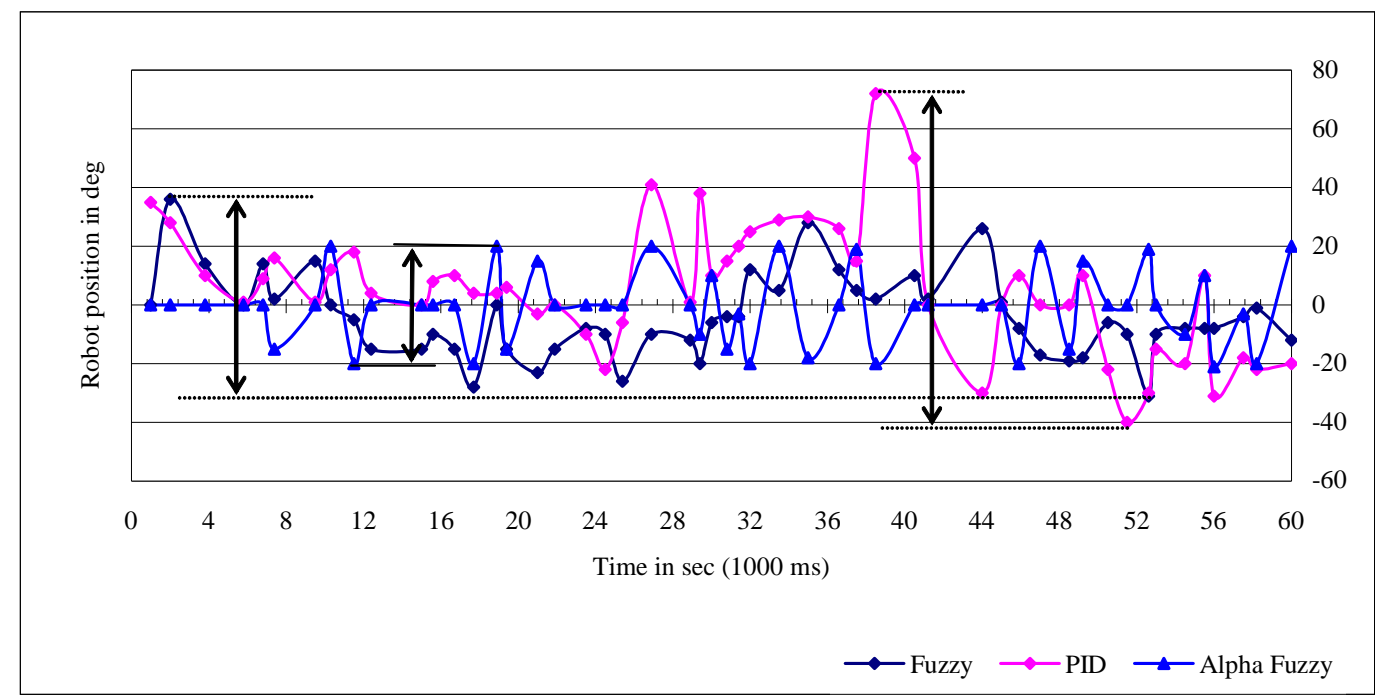

Fig 17. Comparison of results using PID, Fuzzy and Alpha Fuzzy control system.

The robot navigation based on Kalman filtering [23] and [24] requires mathematical models for the system estimation and measuring instruments and the filter called data processing algorithm is a black box containing an electrical network, which processes the inputs and produce outputs. In the proposed method, the most useful measurement data are integrated and mapped onto the system model itself. The model used in the proposed method for the decision making is an integral part of the control process. In the proposed method, the fuzzy algorithm makes a decision by considering several different types of situations at the same time resulting in a more human like decision.

Genetic Algorithm [25] performs randomized global search of an environment whereas the proposed Alpha level fuzzy system uses continuous search. The present methodology is based on classical reasoning and transparent modeling approaches that are invariably based on Boolean logic, which is simple to implement for optimization of robot bath navigation.

\section{CONCLUSION}

A new deterministic approach to resolve the behavior conflicts in the complex situation during mobile robot navigation is developed and validated with real world experiments. The behavior rules containing the Alpha intervals of input and output spaces are adapted 
with-ease and the robot is found to navigate with minimum deviation when encountered with obstacles. The experimental results clearly indicate the mapping of inputs to outputs with optimum path in every control cycle of robot navigation. This approach involves the natural way of dealing with the environments using simple linguistic logic rules without using any mathematical model. The knowledge base of each behavior rule is easy to comprehend, because it captures the behavior rules in a linguistic form by simple intuitive Alpha threshold interval based rule statements.

\section{REFERENCES}

[1] R.A. Brooks, "A Robust Layered Control System for a Mobile Robot," IEEE Journal of Robotics and Automation, Vol. RA-2, No.1, pp 14-23. Year?

[2] R.C. Arkin, "Motor Schema Based Navigation for a Mobile Robot: An Approach to Programming by Behavior," proceedings of the IEEE Intl. Conference on Robotics and Automation, 1987, pg 264-271.

[3] E.Gat, "On the Role of Stored Internal State in the Control of Autonomous Mobile Robots," AI Magazine, pp. 64-73, Spring 1993.

[4] L.T. Anderson and Max Donath, "Animal Behavior as a Paradigm for Developing Robot Autonomy," Robotics and Autonomous Systems, Vol. 6, 1990, pages 145-168.

[5] W.T and Zhang Bo, "Time-Varying (sic) Potential Field Based 'Perception-Action' Behaviors of Mobile Robot," in Proceedings of the 1992 IEEE International Conference on Robotics and Automation, May, 1992, Nice, France

[6] P.Maes, "How To Do the Right Things". NE 43-836, AI-Laboratory, MIT, MA 02139, USA, 1989.

[7] P. Althaus, H. Christiansen, and F.Hoffmann. Using the dynamical system approach to navigate in realistic real-world environments. In proceedings of the IEEE/RSJ International conference on Intelligent robotics and Systems, Page 1023-1029, 2001.

[8] D.C Mackenzie, R.C Arkin, and J.M. Cameron, "Specification and Execution of Multiagent mission," College of computing, Georgia Institute of Technology, Atlanta, Georgia, USA. 1995.

[9] R.C Arkin, "Integrating behavioral, perceptual, and world knowledge in reactive navigation," Robotics and Autonomous Systems 6, pp. 105-122, June 1990.

[10] O.Khatip, Real-time Obstacle Avoidance for Manipulators and Mobile robots", The international journals of Robotics Research 5(1), pp. 90-98,1986.

[11] R.C.Arkin, "Towards the unification of Navigational planning and reactive Control," AAAI Press, 1995.

[12] A.Saffotti, K.Konolige, and E.H Ruspini," A multi-valued logic approach to integrating planning and control," Artificial Intelligence, March 1995.

[13] E.W. large, H.I. Christensen, and R.Bajcsy, "Scaling the dynamic approach to path planning and control: Competition among Behavioral Constrain," International journal of robotics research, 1997.

[14] J.K Rosenblatt, "DAMN: A Distribute Architecture for Mobile Navigation," in AAAI Press, march 1995.

[15] A. Saffiotti, "Fuzzy logic in autonomous robotics: Behavior coordination," in Proc. 6th Int. Conf. Fuzzy Systems, Barcelona, Spain 1997, vol.1, pp. 573-578. 
[16] E. Gat, R. Desai, R. Ivlev, J. Loch, and D.P. Miller, "Behavior control exploration of planetary surfaces,” IEEE. Trans. Robot. Automat., VOL. 10, Aug 1994.

[17] E. Tunnstel, T.Lippincott, and M.Jamshidi, "Behavior hierarchy for autonomous mobile robots: Fuzzy behavior modulation and Evolution," In. J. Intell. Automat. Soft Compute., vol 3, no. 1, pp 37-49, 1997.

[18] S.Parasuraman, V.Ganapathy, Bijan Shirinzadeh, "Behavior based Mobile Robot Navigation by AI Techniques: Behavior selection and resolving behavior conflicts using Alpha level Fuzzy Inference System" Proceeding of the ICGST International conference on Automation, Robotics and Autonomous System, ARAS2005, EGYPT. P1130533120.

[19] Liu Xin; Vadakkepat, P.; Tong Heng Lee; Xiao Peng; Pang Ki Kim. "Comparison of Khepera robot navigation by evolutionary neural networks and pain-based algorithm Evolutionary Computation", 2002. CEC '02. Proceedings of the 2002 Congress on Volume 2, 12-17 May 2002 Page(s): 1994 - 1999.

[20] Petru Rusu, Thom E.Whalen, Aurel Cornell and Hans J.W Spoelder "Behavior based Neuro-Fuzzy Controller for Mobile Robot Navigation IEEE Transaction on Instrumentation and measurement", vol. 52, No. 4, August 2003.

[21] Botelho, S.S.C. do Valle Simoes, E.; Uebel, L.F.; Barone, D. "High speed neural control for robot navigation" Systems, Man, and Cybernetics, 1996. IEEE International Conference on Volume 3, 14-17 Oct. 1996 Page(s):1956 - 1959 vol.3.

[22] Jong-Soo Kim, Seong-Joo Kim and Hong-Tae Jeon "Intelligent Trace Algorithm of Mobile Robot Using Fuzzy Logic" college of Engineering Chung Ang University, Seoul, 156-756, Korea.

[23] Simon D: Kalman Filtering, Embedded.com, viewed on Dec. 10, 2001. http://www.embedded.com/showArticle.jhtml?article ID=9900168 (2001).

[24] Welch, G., Bishop, G.: An Introduction to the Kalman Filter, ACM SIGGRAPH 2001, Course 8, available at http://www.cs.unc.edu/_welch/kalman (2001).

[25] John H. Holland, Adaptation in natural and artificial systems, MIT Press, Cambridge, MA, 1992 . 